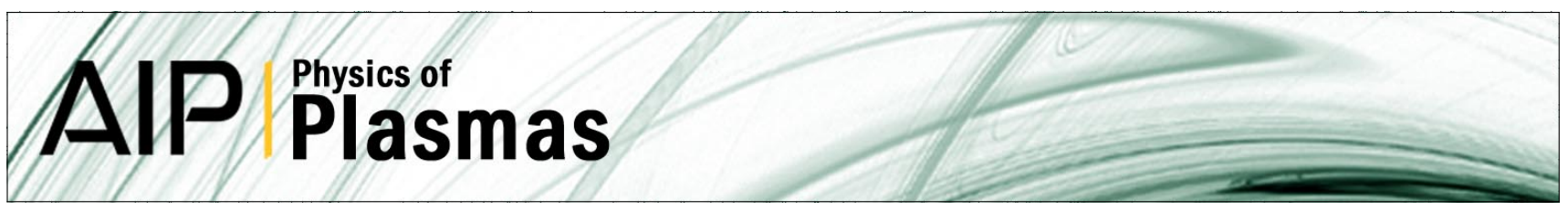

\title{
Plasma turbulence in the scrape-off layer of tokamak devices
}

Paolo Ricci and B. N. Rogers

Citation: Phys. Plasmas 20, 010702 (2013); doi: 10.1063/1.4789551

View online: http://dx.doi.org/10.1063/1.4789551

View Table of Contents: http://pop.aip.org/resource/1/PHPAEN/v20/i1

Published by the American Institute of Physics.

\section{Related Articles}

Roles of poloidal rotation in the $\mathrm{q}=1$ high-order harmonic tearing modes in a tokamak plasma Phys. Plasmas 20, 012512 (2013)

Toroidal precession as a geometric phase

Phys. Plasmas 20, 012511 (2013)

Note: Measurements of fast electrons in the TORE-SUPRA tokamak by means of modified Cherenkov-type diamond detector

Rev. Sci. Instrum. 84, 016107 (2013)

Gyrokinetic simulation of global and local Alfvén eigenmodes driven by energetic particles in a DIII-D discharge Phys. Plasmas 20, 012508 (2013)

Interpretation of changes in diffusive and non-diffusive transport in the edge plasma during pedestal buildup following a low-high transition in DIII-D

Phys. Plasmas 20, 012509 (2013)

\section{Additional information on Phys. Plasmas}

Journal Homepage: http://pop.aip.org/

Journal Information: http://pop.aip.org/about/about_the_journal

Top downloads: http://pop.aip.org/features/most_downloaded

Information for Authors: http://pop.aip.org/authors

\section{ADVERTISEMENT}

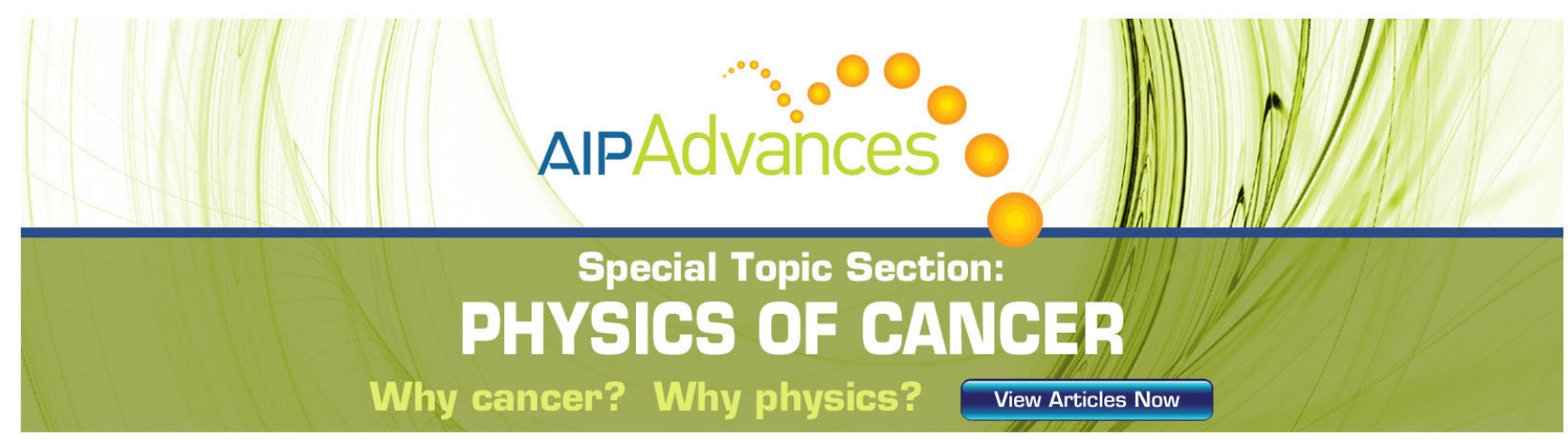




\title{
Plasma turbulence in the scrape-off layer of tokamak devices
}

\author{
Paolo Ricci ${ }^{1, \text { a) }}$ and B. N. Rogers ${ }^{2, b)}$ \\ ${ }^{1}$ Centre de Recherches en Physique des Plasmas - École Polytechnique Fédérale de Lausanne, \\ Association EURATOM-Confédération Suisse, CH-1015 Lausanne, Switzerland \\ ${ }^{2}$ Department of Physics and Astronomy, Dartmouth College, Hanover, New Hampshire 03755, USA
}

(Received 22 November 2012; accepted 9 January 2013; published online 28 January 2013)

Plasma turbulence is explored in the scrape-off layer of tokamak devices using three-dimensional global two-fluid simulations. Two transport regimes are discussed: one in which the turbulent fluctuations saturate nonlinearly due to the Kelvin-Helmholtz instability, and another in which the fluctuations saturate due to a local flattening of the plasma gradients and associated removal of the linear instability drive. Focusing on the latter regime, analytical estimates of the cross-field transport and plasma profile gradients are obtained that display Bohm-scaling diffusion properties. [http://dx.doi.org/10.1063/1.4789551]

Plasma dynamics in the edge of magnetic fusion devices largely govern the performance of the entire device. ${ }^{1}$ Of key interest is the scrape-off layer (SOL) region, in which the magnetic field lines are open and terminate on the machine vessel. The plasma dynamics in this region, which involve the interplay between the plasma fluxes from the core region, the turbulent transport across the magnetic field lines, and the losses to the vessel walls, determine the peak heat loads at the vessel-one of the most critical issues in fusion reactors. A widely accepted theoretical model of the SOL region remains elusive, compromising our ability to reliably predict the performance of future devices such as ITER.

In this Letter, we present three-dimensional two-fluid simulations of plasma turbulence in the tokamak SOL. Using an approach similar to the simulations in, e.g., Refs. 2 and 3, we evolve the full profiles of the various quantities with no separation made between perturbations and equilibrium, and therefore we explore the self-consistent evolution and structure of the plasma profiles in the presence of (i) the plasma and heat flowing from the tokamak core to the SOL, (ii) the cross-field transport produced by plasma turbulence, (iii) plasma flow along the field lines, and (iv) plasma losses at the sheaths where the magnetic field lines terminate on the walls. We focus on a relatively simple configuration: circular magnetic flux surfaces and a toroidal limiter placed on the high-field side equatorial midplane of the device, with negligible $E \times B$ shear flow. This relative simplicity allows analytical progress and the identification of the mechanisms that presumably underlie the physics of more complicated configurations. In fact, we derive analytic estimates of the SOLturbulence saturation level, perpendicular transport, plasma profile scale-lengths, and other quantities that are in reasonable agreement with the simulations over a wide range of parameters. These indicate the turbulent fluctuations are roughly comparable to the time-averaged quantities, develop across a radial zone that is intermediate between the macroscopic equilibrium scale length and the microscopic scale

\footnotetext{
${ }^{\text {a)}}$ Electronic mail: paolo.ricci@epfl.ch.

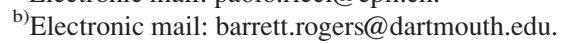

length of linear modes, and lead to Bohm-type transport scaling.

The SOL simulations evolve the drift-reduced Braginskii equations (see, e.g., Refs. 4 and 5) in a limit that can capture the resistive-ballooning dynamics, while remaining relatively simple: $T_{i} \ll T_{e}$, large aspect ratio, and circular flux surfaces. The main results reported here have been observed in simulations over a range of magnetic shear and $\beta$ values $\left(|\hat{s}| \lesssim 3, \alpha=R q^{2} \beta^{\prime} \lesssim 0.1\right.$ ), but most simply from an electrostatic model with $\hat{s}=0$

$$
\begin{gathered}
\frac{d n}{d t}=\frac{2 c}{e B}\left[\hat{C}\left(p_{e}\right)-e n \hat{C}(\phi)\right]-\frac{\partial\left(n V_{\| e}\right)}{\partial z}+S_{n} \\
\frac{d \nabla_{\perp}^{2} \phi}{d t}=\frac{2 B}{c n m_{i}} \hat{C}\left(p_{e}\right)-V_{\| i} \frac{\partial \nabla_{\perp}^{2} \phi}{\partial z}+\frac{m_{i} \Omega_{c i}^{2}}{e^{2} n} \frac{\partial j_{\|}}{\partial z} \\
\frac{d T_{e}}{d t}=\frac{4}{3} \frac{c}{e B}\left[\frac{7}{2} T_{e} \hat{C}\left(T_{e}\right)+\frac{T_{e}^{2}}{n} \hat{C}(n)-e T_{e} \hat{C}(\phi)\right] \\
+\frac{2}{3} \frac{T_{e}}{e n} 0.71 \frac{\partial j_{\|}}{\partial z}-\frac{2}{3} T_{e} \frac{\partial V_{\| e}}{\partial z}-V_{\| e} \frac{\partial T_{e}}{\partial z}+S_{T} \\
m_{e} \frac{d V_{\| e}}{d t}=-m_{e} V_{\| e} \frac{\partial V_{\| e}}{\partial z}-\frac{T_{e}}{n} \frac{\partial n}{\partial z}+e \frac{\partial \phi}{\partial z}-1.71 \frac{\partial T_{e}}{\partial z}+\frac{e j_{\|}}{\sigma_{\|}} \\
\frac{d V_{\| i}}{d t}=-V_{\| i} \frac{\partial V_{\| i}}{\partial z}-\frac{1}{n} \frac{\partial p_{e}}{\partial z}
\end{gathered}
$$

In Eqs. (1)-(5), $\quad p_{e}=n T_{e}, \hat{C}=-\sin (y / a) \partial_{x}$ $-\cos (y / a) \partial_{y}, \quad \nabla_{\perp}^{2}=\partial_{x}^{2}+\partial_{y}^{2}, \quad d f / d t=\partial_{t} f-(c / B)[\phi, f]$, $[f, g]=\partial_{x} f \partial_{y} g-\partial_{y} f \partial_{x} g, \omega=\nabla_{\perp}^{2} \phi, j_{\|}=e n\left(V_{\| i}-V_{\| e}\right), \Omega_{c i}$ $=e B /\left(m_{i} c\right), a$ is the minor radius, $x$ is the radial coordinate, $z$ is parallel to $B, y$ is perpendicular to both $x$ and $z$ (equivalent at large aspect ratio to a poloidal coordinate) with $y=0$ and $y=2 \pi a=L_{y}$ corresponding to the limiter plates at the high-field side equatorial midplane. The source terms $S_{n}=S_{n 0} \exp \left[-\left(x-x_{S}\right)^{2} / \lambda_{S}^{2}\right], \quad S_{T}=S_{T 0} \exp \left[-\left(x-x_{S}\right)^{2} / \lambda_{S}^{2}\right]$ mimic the plasma flowing from the tokamak core and thus the $x>x_{S}$ region is one of the interest in our simulations (we neglect ionization, recombination, and radiative losses). 
Here, we present simulations with $\lambda_{S}=5 \rho_{s}$ though we have checked that our main findings do not significantly depend on this parameter. We consider Eqs. (1)-(5) in a domain that covers the full toroidal angle, extends radially from $x=0$ to $x=L_{x}$ and poloidally from $y=0$ to $y=L_{y}$. Neumann boundary conditions are applied along the $x$ direction. The plasma sheath losses are implemented at the limiter plates, $y=0$ and $y=2 \pi a$, by using Bohm's boundary conditions for the parallel velocities, $V_{\| i}= \pm c_{s}$ and $V_{\| e}= \pm c_{s} \exp \left(\Lambda-e \phi / T_{e}\right)$, while Dirichelet boundary conditions are imposed for the other fields. Simulations with other sets of both perpendicular and parallel boundary conditions, as well as other limiter positions, suggest that these elements do not impact our main results. Equations (1)-(5) are solved by the GBS code $e^{5}$ using a finite difference scheme with Runge-Kutta time stepping. Small numerical diffusion terms are added, as described in Ref. 5.

Simulations have been performed over a range of parameters, including the safety factor $q=(2,4,8,16)$, $\nu=e^{2} n /\left(m_{i} \sigma_{\|}\right)=(0.01,0.1,1,6) c_{s} / R, m_{e} / m_{i}=200, L_{y}$ $=(200,400,800) \rho_{s}, R=500 \rho_{s}$. The strengths of the density and temperature sources have been varied by a factor of four. The simulations are started with small amplitude random noise; the sources then introduce plasma and heat, increasing the plasma pressure and triggering curvature and pressure-gradient driven instabilities. After a transient phase, a quasi-stationary state is reached in which the plasma, generated by the source and transported by the turbulent dynamics in the radial and parallel directions, is eventually removed from the system by the losses at the limiter plates. Fig. 1 shows a snapshot of plasma turbulence in this quasistationary state, revealing the presence of plasma blobs, a prominent feature of the SOL dynamics. ${ }^{7}$

Turbulence in our system stems principally from the resistive ballooning mode and, depending on the simulation parameters, drift waves (DWs) can also be active. Resistive ballooning modes have maximum growth rates comparable to the ideal interchange mode $\gamma^{2} \sim c_{s}^{2} /\left(R L_{p}\right)=\gamma_{b}^{2}$ and occur
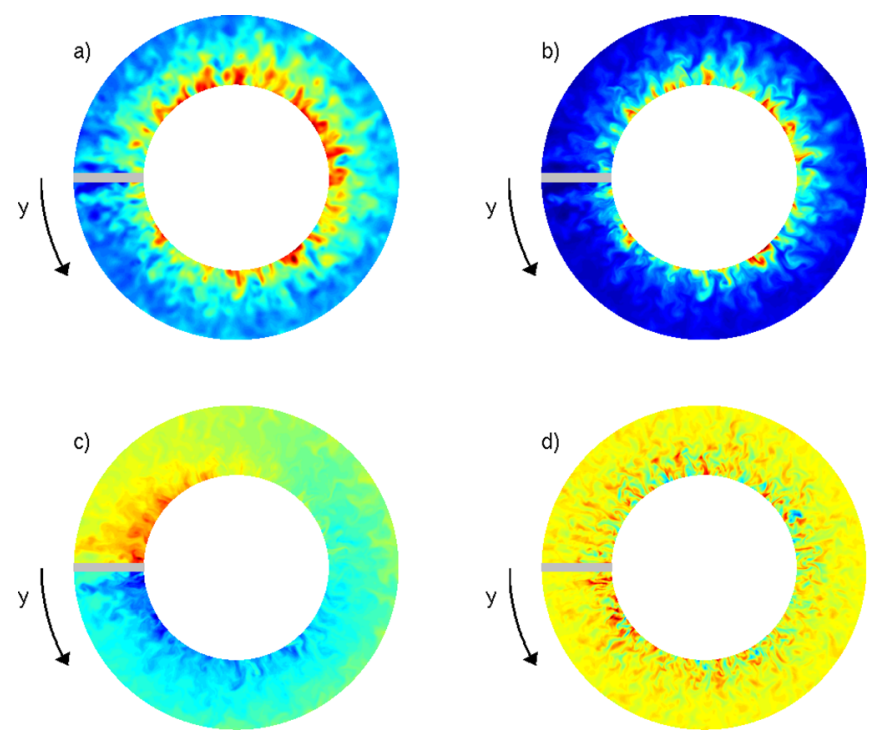

FIG. 1. Typical snapshot of plasma turbulence: $\phi$ (a), $p_{e}$ (b), $n V_{\| i}$ (c), and $j_{\|}$ (d) are displayed on a poloidal cross section. It is $q=4, \nu=0.01, L_{y}=800$. when the resistivity is sufficiently high. The instability threshold follows from the vorticity equation [Eq. (2)]: the polarization drift term $\partial_{t} \nabla_{\perp}^{2} \phi$ must exceed the line bending term proportional to $\nabla_{\|} j_{\|}$; with Ohm's law [Eq. (4)], $j_{\|} \sim \sigma_{\|} \nabla_{\|} \phi$, this condition yields $k_{\perp}^{2}>4 \pi V_{A}^{2} k_{\|}^{2} \sigma_{\|} /\left(\gamma_{b} c^{2}\right)$ $=k_{b}^{2}$. On the other hand, when gradients become sufficiently steep, DW can play a role. DW have peak growth rate, $\gamma \sim c_{s} / L_{p}$, in correspondence of $k_{y} \rho_{s} \sim 1 .^{6}$

Through our simulations, we explore two mechanisms that saturate the growth of the linear modes. The first is the Kelvin-Helmholtz $(\mathrm{KH})$ instability, which is excited by the radial streamers and associated velocity shear arising from the primary instabilities. It is typically triggered when the nonlinear and linear terms in the vorticity equation become competitive, $\partial_{t} \omega \sim(c / B)[\phi, \omega]$, leading to $\tilde{\phi} \sim B \gamma /\left(c k_{x} k_{y}\right)$ (we denote equilibrium, time-averaged quantities with an overbar, and fluctuating quantities with a tilde, e.g., $\phi=\bar{\phi}+\tilde{\phi})$. The pressure fluctuations can be estimated from a similar balance in the continuity and temperature equations [Eqs. (1) and (3)] as $\partial_{t} p_{e} \sim(c / B)\left[\phi, p_{e}\right]$ or $\gamma \tilde{p}_{e} \sim c k_{y} \bar{p}_{e} \tilde{\phi} /\left(B L_{p}\right)$, or with the previous estimate for $\tilde{\phi}$ : $\tilde{p}_{e} / \bar{p}_{e} \sim 1 /\left(k_{x} L_{p}\right)$. Assuming $L_{n} \sim L_{T} \sim L_{p}$, the radial heat flux, $\Gamma_{x}=(c / B) \tilde{p}_{e} \partial_{y} \tilde{\phi}$, is therefore $\Gamma_{x} \sim \bar{p}_{e} \gamma /\left(L_{p} k_{x}^{2}\right)$, and the associated anomalous diffusion coefficient $D=\Gamma_{x} L_{p} / \bar{p}_{e} \sim \gamma / k_{x}^{2}$. Finally, since the KH instability has a peak growth rate at $k_{x} \sim k_{y}$, we are led, assuming $\mathrm{KH}$ is dominant in the saturation mechanism for the turbulence, to the usual mixing length result, $D_{K H}=\gamma / k_{y}^{2}$, for the anomalous diffusion coefficient. In the standard reference-case that DWs are the dominant primary instability, this gives a Gyrobohm diffusion coefficient $D \sim \rho_{s} c_{s}\left(\rho_{s} / L_{p}\right)$.

In addition to the KH instability, a second possible turbulence saturation mechanism present in the simulations is the local nonlinear flattening of the plasma gradients by the primary modes, which requires (for example) $\partial_{x} \bar{p}_{e} \sim \partial_{x} \tilde{p}_{e}$. Following nonlocal linear theory methods (outlined, e.g., in Ref. 8 for DW and Ref. 9 for curvature-driven modes), one can estimate the typical radial extension of the linear modes present in the SOL in usual turbulence regime of $k_{y} L_{p}>1$ and within the hypothesis, satisfied in our simulations, that the pressure gradient varies on a spatial scale comparable to $L_{p}$. For both DW and ballooning modes, one finds $k_{x} \sim \sqrt{k_{y} / L_{p}}$, an intermediate scale between the turbulence and equilibrium scale lengths. With the hypothesis that the radial width of the modes is carried over by the turbulent eddies, one therefore obtains a characteristic amplitude for the pressure fluctuations $\tilde{p}_{e} / \bar{p}_{e} \sim 1 / \sqrt{k_{y} L_{p}}$. With the relation between $\tilde{\phi}$ and $\tilde{p}_{e}$ obtained earlier from the pressure equation, the estimates for the heat flux and anomalous diffusion coefficient, assuming the gradient removal is the mechanism at play, are $\Gamma_{x} \sim \bar{p}_{e} \gamma / k_{y}$ and $D_{G R}=\gamma L_{p} / k_{y}$. In the case of DW, this gives a Bohm diffusion coefficient $D \sim \rho_{s} c_{s}$.

Which of the two saturation mechanisms are at play in the SOL? Comparing the diffusion coefficients, one finds $D_{K H} / D_{G R} \sim 1 /\left(k_{y} L_{p}\right)<1$. Provided that $\mathrm{KH}$ is unstable, we would therefore expect it to dominate, since it will act first to limit the growth of the linear modes and therefore the transport to smaller levels than the gradient-removal mechanism. But this prediction is not always consistent with the 

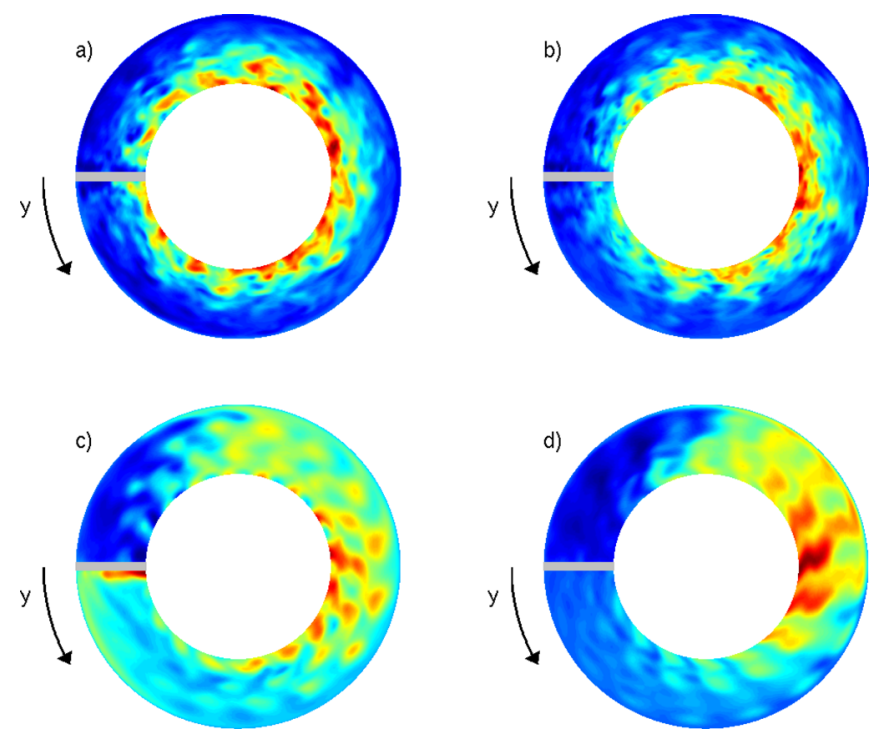

FIG. 2. Effect of removing the KH instability from the simulations. A $q=4$ simulation is considered in (a) and (b) panels, while $q=16$ in (c) and (d). We replace $[\omega, \phi] \rightarrow\left[\omega,\langle\phi\rangle_{y}\right]$ in (b) and (d). We display $\phi$ on a poloidal cross section.

simulations. To explore this, we removed the $\mathrm{KH}$ instability from the simulations by replacing $\phi$ in the Poisson bracket of Eq. (2) with its instantaneous $y$-average, $\langle\phi\rangle_{y}$, i.e., $[\omega, \phi] \rightarrow\left[\omega,\langle\phi\rangle_{y}\right]$. Fig. 2 shows typical snapshots in the presence and absence of the KH term in a $q=4$ and $q=16$ simulations. Contrary to our prediction, in the case of the $q=4$ simulation, there are only small changes when the $\mathrm{KH}$ term is turned off, e.g., $L_{p}$ remains approximatively the same, suggesting that $\mathrm{KH}$ does not play a fundamental role in the simulations. In the $q=16$ simulation, however, as is typically the case in high- $q$ simulations, the turbulent eddies become elongated when the $\mathrm{KH}$ is removed from the system, and a flattening of the profile is observed, i.e., $L_{p}$ doubles. This suggests the KH instability is indeed responsible for the saturation process in some cases (such as high q) but not in others.

To explain this, we note that the peak growth rate of the $\mathrm{KH}$ instability for a sinusoidal $E \times B$ flow in the $x$ direction, $V_{E}=V_{0} \sin \left(k_{y} y\right) \mathbf{e}_{x}$, is given by $\gamma_{K H} \simeq 0.3 k_{y} V_{0}$ and occurs at $k_{x} \simeq 0.6 k_{y}{ }^{8}$ We also note that the turnover time of an eddy flow with $x$ extension $\sigma_{x}$ is given by $\tau_{e d d y} \sim \sigma_{x} / V_{0}$. For the $\mathrm{KH}$ mode to grow, one would expect $\gamma_{K H} \tau_{e d d y}>1$ to be necessary. We have confirmed this threshold in two dimensional simulations of the vorticity equation, $d \nabla_{\perp}^{2} \phi / d t=0$, considering as initial condition eddies with different elongations: elongated eddies with $\gamma_{K H} \tau_{e d d y}>1$ are destroyed by the $\mathrm{KH}$ instability, while $\gamma_{K H} \tau_{e d d y}<1$ eddies are not affected and persist in time. For the turbulent eddies $\sigma_{x} \sim \sqrt{L_{p} / k_{y}}$ and $\tau_{\text {eddy }} \sim \sqrt{L_{p} /\left(k_{y} V_{0}^{2}\right)}$, therefore, $\gamma_{K H} \tau_{e d d y} \sim 0.3 \sqrt{k_{y} L_{p}}$. We therefore expect that for simulations characterized by $\sqrt{k_{y} L_{p}}<3, \mathrm{KH}$ is stable, and the gradient removal process leads to turbulence saturation. In Fig. 3(a), we plot the $k_{y}$ value of the typical turbulent eddies dominating various simulations versus $\sqrt{L_{p} / k_{y}}$, also noting the cases in which, according to our numerical $\mathrm{KH}$ shut-off tests, $\mathrm{KH}$ plays an essential role. In agreement with our estimate, the $\mathrm{KH}$ saturated simulations are characterized by $\sqrt{k_{y} L_{p}}>3$.

In the present Letter, we focus on the dynamics of the $\mathrm{KH}$-stable cases. To test if the gradient removal is the saturation mechanism that operates in these cases, in Fig. 3(b) we show, for the $\sqrt{k_{y} L_{p}}<3$ simulations, that the radial width of the eddies, $\sigma_{x}$, scales according to the gradient-removal estimate, $\sqrt{L_{p} / k_{y}}$. Moreover, according to our heat-flux estimate in the case of gradient-removal, $\Gamma_{x} \sim \bar{p}_{e} \gamma / k_{y}$, we
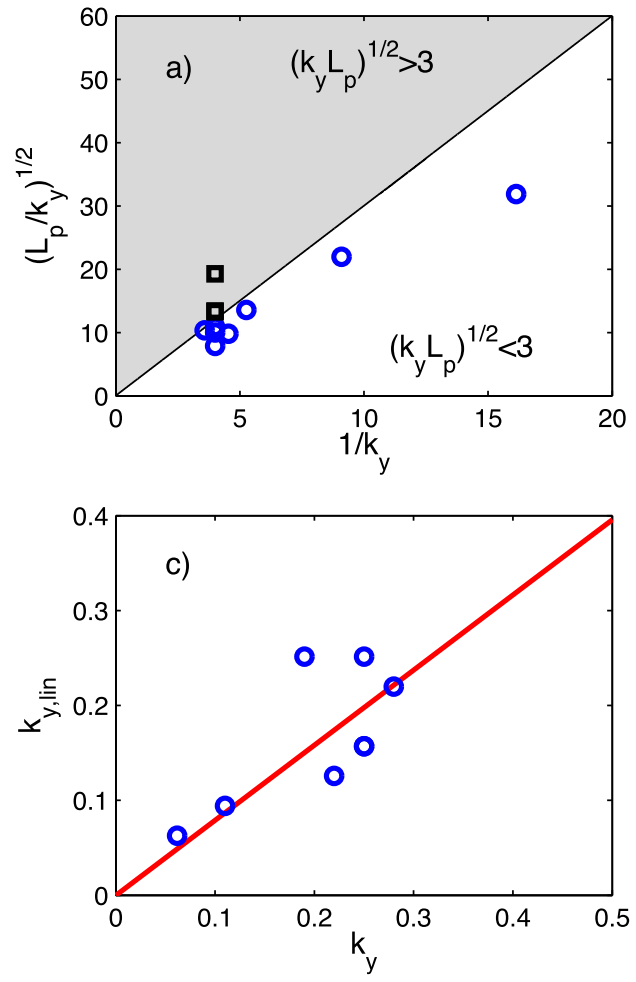
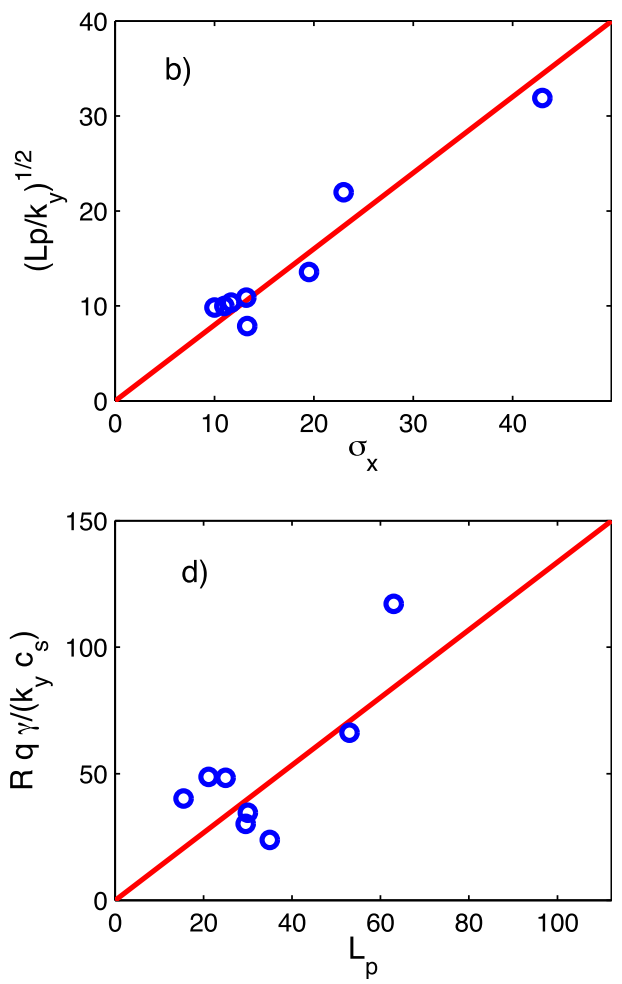

FIG. 3. $\sqrt{L_{p} / k_{y}}$ vs $1 / k_{y}$ (a). Black squares represent simulations where $\mathrm{KH}$ sets the turbulence amplitude, blue circles indicate simulations where removing the KH term does not lead to substantial changes in the turbulence. For the second set of simulations, we show the comparison of the eddy radial width versus the gradient-removal estimate (b), of the maximum-amplitude mode $k_{y}$ versus the $k_{y}$ of the mode with maximum $\gamma / k_{y}$ (c), and of the pressure scale length versus the gradient-removal estimate (d). Lengths are normalized to $\rho_{s}$. 
expect that the transport is dominated by the mode with the highest $\gamma / k_{y}$. In Fig. 3(c), we show the comparison between the $k_{y}$ of the mode that dominates the spectrum of $\phi$ (or $p_{e}$ or $n$ ) in the simulations and the $k_{y}$ of the mode corresponding to the highest value of $\gamma / k_{y}$, as obtained from the linear mode analysis carried out for each simulation. The good agreement suggests that gradient removal is indeed the mechanism at play in the KH-stable simulations.

With this understanding of saturation mechanism, we now analyze the scaling of the plasma profiles. Assuming that parallel losses dominate at the limiter plates and, according to Bohm's criterion, can be expressed as $p_{e} c_{s}$, ${ }^{10}$ one can time and flux-surface average the leading order terms for the density and temperature equations, obtaining $d\left\langle\Gamma_{x}\right\rangle_{y} / d x$ $\sim \bar{p}_{e} \bar{c}_{s} /(q R)$ in the source-free region. Approximating $d\left\langle\Gamma_{x}\right\rangle_{y} /$ $d x \sim\left\langle\Gamma_{x}\right\rangle_{y} / L_{p}$ and using the estimate $\left\langle\Gamma_{x}\right\rangle_{y} \sim \gamma \bar{p}_{e} / k_{y}$, one obtains the pressure scale length $L_{p} \sim R q \gamma /\left(k_{y} c_{s}\right)$, which is intermediate between macroscopic and turbulent scale lengths. The comparison between this $L_{p}$ scaling and the simulation results is shown in Fig. 3(d).

Assuming the ballooning mode is the dominant instability, consistent with our simulations as well as experimental observations in the far SOL, ${ }^{11}$ we can now obtain a selfconsistent estimate of the plasma scale length in the SOL. Assuming $\gamma \sim \gamma_{b}$, one obtains $L_{p} \sim R^{1 / 3}\left(q / k_{y}\right)^{2 / 3}$. Moreover, one expects (from the peak of $\gamma / k_{y}$, for example) that $k_{y} \sim k_{b}$, where $k_{b}$ is the typical resistive ballooning mode wavenumber given earlier. Substituting $k_{\|} \sim 1 /(q R)$ into $k_{b}$ finally leads to $L_{p} \sim R^{5 / 7} q^{8 / 7}\left[\sqrt{2} c_{s} c^{2} /\left(4 \pi \sigma_{\|} V_{A}^{2}\right)\right]^{2 / 7}$, which favorably predicts a rather broad SOL for large, ITER-scale machines. With this and other estimates, the KH-stable parameter regime is $\sqrt{k_{y} L_{p}} \sim\left[4 \pi \sigma_{\|} V_{A}^{2} R q^{3} /\left(\sqrt{2} c_{s} c^{2}\right)\right]^{1 / 14}<3$, which is consistent with the linkage in our simulations of high- $q$ and saturation due to the $\mathrm{KH}$ instability.

Accurate measurements have been carried in the SOL of the Alcator C-Mod tokamak, using standard Langmuir probes and gas puff imaging. In Ref. 12, an inner-wall limited, nearly circular Ohmic plasma is described, similar to the scenario analyzed herein. The measurement data show across the SOL that $L_{p} \simeq 1 \mathrm{~cm}$ and $L_{n} \simeq 2 \mathrm{~cm}$ (profiles are steeper closer to the last close flux surface and less steep in the far SOL), while the correlation length in the poloidal direction shows $k_{y} \simeq 2.5 \mathrm{~cm}^{-1}$. In particular, it is observed that $\sqrt{k_{y} L_{p}} \simeq 1.5$; therefore, we expect that turbulence is saturated by the gradient removal mechanism. In fact, the radial eddy extension is $\sigma_{x} \simeq 1 \mathrm{~cm}$ in agreement with our estimate, $\sigma_{x} \sim \sqrt{L_{p} / k_{y}} \simeq 0.65 \mathrm{~cm}$. The relative fluctuation level of light emission in the experiment is about $40 \%$, the same as our prediction $\tilde{p}_{e} / \bar{p}_{e} \sim 1 / \sqrt{k_{y} L_{p}} \simeq 0.6$. We note that also in the TCV tokamak ${ }^{13}$ and in other tokamaks (see Ref. 14 for a review), fluctuations of order unity have been observed in the SOL, a higher value than expected in the case of KH saturation. Regarding the estimate of the plasma scalelength, based on $\gamma \sim \gamma_{b}$, one obtains $L_{p} \sim R^{1 / 3}\left(q / k_{y}\right)^{2 / 3}$ $\simeq 4 \mathrm{~cm}$ which is in reasonable agreement with the measurement in the far SOL measurements.

In conclusion, in the present Letter, we have carried out an analysis of turbulence in the SOL in a relatively simple scenario, revealing the mechanisms that set the amplitude of the turbulence. This has led to estimates of cross-field transport, plasma scale lengths, and other quantities. In the typical case, that the gradient-removal mechanism is at play in setting the turbulence level, the transport displays Bohm scaling in the canonical setting of DW and a related scaling for resistive ballooning modes. We note that Bohm-diffusion scalings are pointed out by experimental results in the SOL (see Ref. 10 for a discussion), and the claim has been made also in the core (see, e.g., Ref. 15). As in simpler plasma geometries, ${ }^{6}$ our analysis underscores the importance of simulating the full evolution of the plasma profiles without separation between the fluctuation and equilibrium scales. Further investigations are needed in order to extend our analysis to the description of diverted tokamak where experimental observations suggest a size-independent scaling for $L_{p}{ }^{16}$

This work was supported by the Swiss National Science Foundation and the US-DOE through the CICART grant at Dartmouth. We acknowledge many useful discussions with A. Fasoli, I. Furno, F. Halpern, S. Jolliet, J. Loizu, and A. Mosetto.

${ }^{1}$ A. Loarte, B. Lipschultz, A. S. Kukushkin, G. F. Matthews, P. C. Stangeby, N. Asakura, G. F. Counsell, G. Federici, A. Kallenbach, K. Krieger, A. Mahdavi et al., Nucl. Fusion 47, S203 (2007).

${ }^{2}$ X. Q. Xu, R. H. Cohen, T. D. Rognlien, and J. R. Myra, Phys. Plasmas 7, 1951 (2000).

${ }^{3}$ T. T. Ribeiro and B. Scott, Plasma Phys. Controlled Fusion 50, 055007 (2008).

${ }^{4}$ A. Zeiler, J. F. Drake, and B. Rogers, Phys. Plasmas 4, 2134 (1997).

${ }^{5}$ P. Ricci, F. Halpern, S. Jolliet, J. Loizu, A. Mosetto, A. Fasoli, I. Furno, and C. Theiler, Plasma Phys. Controlled Fusion 54, 124047 (2012).

${ }^{6}$ B. N. Rogers and P. Ricci, Phys. Rev. Lett. 104, 225002 (2010); P. Ricci and B. N. Rogers, ibid. 104, 145001 (2010).

${ }^{7}$ D. A. D’Ippolito, J. R. Myra, and S. J. Zweben, Phys. Plasmas 18, 060501 (2011).

${ }^{8}$ B. N. Rogers and W. Dorland, Phys. Plasmas 12, 062511 (2005).

${ }^{9}$ P. Ricci, B. N. Rogers, and S. Brunner, Phys. Rev. Lett. 100, 225002 (2008); P. Ricci and B. N. Rogers, Phys. Plasmas 16, 062303 (2009).

${ }^{10}$ P. C. Stangeby, The Plasma Boundary of Magnetic Fusion Devices (Institute of Physics Publishing, 2000).

${ }^{11}$ B. LaBombard, J. W. Hughes, D. Messessian, M. Greenwald, B. Lipschultz, and J. L. Terry, Nucl. Fusion 45, 1658 (2005).

${ }^{12}$ S. J. Zweben, B. D. Scott, J. L. Terry, B. LaBombard, J. W. Hughes, and D. P. Stotler, Phys. Plasmas 16, 082505 (2009).

${ }^{13}$ O. E. Garcia, J. Horacek, R. A. Pitts, A. H. Nielsen, W. Fundamenski, J. P. Graves, and V. Naulin, Plasma Phys. Controlled Fusion 48, L1 (2006).

${ }^{14}$ S. J. Zweben, J. A. Boedo, O. Grulke, C. Hidalgo, B. LaBombard, R. J. Maqueda, P. Scarin, and J. L. Terry, Plasma Phys. Controlled Fusion 49, S1 (2007).

${ }^{15}$ X. Garbet and R. E. Waltz, Phys. Plasmas 3, 1898 (1996).

${ }^{16}$ T. Eich, B. Sieglin, A. Scarabosio, W. Fundamenski, R. J. Goldston, A. Herrmann, and the ASDEX Upgrade Team, Phys. Rev. Lett. 107, 215001 (2011). 